\title{
Epigenetic contributions in the development of rheumatoid arthritis
}

\author{
Kerstin Klein*, Caroline Ospelt and Steffen Gay
}

\begin{abstract}
Rheumatoid arthritis (RA) is an autoimmune disease, characterized by chronic inflammation of the joints with severe pain and swelling, joint damage and disability, which leads to joint destruction and loss of function. Despite extensive research efforts, the underlying cause for RA is still unknown and current therapies are more or less effective in controlling symptoms but still fail to cure the disease. In recent years, epigenetic modifications were found to strongly contribute to the development of RA by affecting diverse aspects of the disease and modifying gene expression levels and behavior of several cell types, first and foremost joint resident synovial fibroblasts (SF). RASF are the most common cell type at the site of invasion. Owing to their aggressive, intrinsically activated phenotype, RASF are active contributors in joint damage. RASF are characterized by their ability to secrete cytokines, chemokines and joint-damaging enzymes. Furthermore, these cells are resistant to apoptosis, leading to hyperplasia of the synovium. In addition, RASF have invasive and migratory properties that could lead to spreading of the disease to unaffected joints. Epigenetic modifications, including DNA methylation and post-translational histone modifications, such as histone (de)acetylation, histone methylation and histone sumoylation were identified as regulatory mechanisms in controlling aggressive cell activation in vitro and in disease outcome in animal models in vivo. In the last 5 years, the field of epigenetics in RA has impressively increased. In this review we consider the role of diverse epigenetic modifications in the development of RA, with a special focus on epigenetic modifications in RASF.
\end{abstract}

Rheumatoid arthritis (RA) is a chronic inflammatory disease affecting about $1 \%$ of the population. Both genetic predisposition and environmental factors, as well as an aberrant activation of the innate and adapted immune system, play a role in the pathogenesis. The major pathological phenomena of RA are the massively elevated levels of inflammatory cytokines produced by activated B cells and $\mathrm{T}$ cells, as well as other cell populations and a synovial hyperplasia, resulting in damage of the cartilage and bone. Characteristically, peripheral joints are often affected in a symmetric distribution. During active disease states, RA leads to swelling and pain, resulting in disability, joint destruction and, ultimately, loss of function. A number of characteristics - such as the occurrence of erosions at the time of presentation shortly after onset of symptoms, synovial infiltrates in clinically unaffected joints and the appearance of autoantibodies long before the onset of the disease - suggest that the disease develops long before clinical symptoms become apparent [1]. At least two subsets of RA exist, which are divided by the presence or absence of antibodies against citrullinated peptide antigens (ACPA). The presence or absence of ACPA has been proven one of the best clinical predictors for disease outcome. Recent genome-wide association studies suggested that ACPA-positive RA and ACPAnegative RA also differ significantly in the frequency of risk alleles. The most important genetic risk factors accounting for 30 to $50 \%$ of the overall risk for RA are mainly confined to the human leukocyte antigen locus [2].

In recent years, knowledge about pathogenic mechanisms and treatment strategies in RA has significantly improved. Only one-third of patients respond well to the first-line treatment methotrexate [3], however, and although the application of biological drugs including therapeutic antibodies directed against TNF $\alpha$ is of great benefit for affected patients, all currently available therapies are more or less effective in controlling symptoms and there is still a subgroup of nonresponding patients. No cure for the disease so far exists and the entire cause of the disease remains elusive.

In the last years, resident synovial fibroblasts (SF) emerged as key players in the development of RA that *Correspondence: kerstin.klein@usz.ch
Center of Experimental Rheumatology, University Hospital Zurich and Zurich
Center of Integrative Human Physiology, Gloriastrasse 23, CH-8091 Zurich,
Switzerland 
actively contribute to joint destruction. RASF are the most common cell type at sites of invasion. By implanting healthy human cartilage together with RASF into severe combined immunodeficient mice, Lefèvre and colleagues demonstrated that activated RASF migrate in vivo and spread the disease to sites of implanted cartilage. Whereas RASF actively degraded human cartilage, control implanted SF from osteoarthritis (OA) patients and dermal fibroblasts from healthy donors did not. Since RASF were able to destroy cartilage even in the absence of cellular and humoral immune responses, this study revealed that RASF are capable of maintaining their activated phenotype without further stimulation of the inflammatory environment in the synovium [4]. RASF differ from healthy synovial fibroblasts by their morphology and an aberrant gene expression pattern [5]. RASF are characterized by the expression of antiapoptotic molecules, protooncogenes and a lack of expression of tumor suppressor genes. Owing to their ability to produce proinflammatory cytokines and chemokines, RASF further attract inflammatory cells of the immune system to the synovium. In addition, RASF produce enzymes such as matrix metalloproteinases (MMPs) that promote invasion into and destruction of cartilage [5]. In recent years RASF were also characterized by aberrant epigenetic modifications that might explain their intrinsic activated phenotype (Figure 1), and in the future they might provide the missing link between RA, risk factors and a lack of therapy response.

Originally, epigenetic modifications were described as heritable alterations of gene expression or cellular phenotypes that were caused by mechanisms other than changes in the DNA sequence. In a more revised definition of epigenetics, also environmentally induced and shorter lived, reversible alterations of the histone code were included [6]. Epigenetic modifications determine the stability of the chromatin structure, the genome integrity, the modulation of gene expression, embryonic development, genomic imprinting and $\mathrm{X}$-chromosome inactivation (XCI) in females [7]. Beside DNA methylation, large networks of post-translational modifications on histone tails, such as acetylation, phosphorylation, methylation, ubiquitination or sumoylation, appear to represent different regulatory mechanisms of chromatin activity in several biological settings [8]. In this review we draw a detailed picture for the role of diverse epigenetic modifications in the development of RA with a special focus on epigenetic modifications in RASF.

\section{DNA methylation}

DNA methylation is the best understood epigenetic modification and in general is associated with gene silencing. DNA methylation is catalyzed by DNA methyltransferases (DNMTs) that use $S$-adenosyl-L-methionine as a methyl donor [7]. The predominant DNMT in somatic cells is DNMT1. In mammalian genomic DNA, position 5 of the cytosine residue within a cytosine-phosphate-guanine dinucleotide $(\mathrm{CpG})$ motif is methylated, resulting in the formation of 5-methylcytosine [7]. Genomic DNA methylation is found throughout the genome. Most $\mathrm{CPG}$ islands, short $\mathrm{CPG}$-rich regions, are generally unmethylated while the majority of $\mathrm{CpG}$ sites in non-CpG islands are methylated [9]. CpG islands are often located in the vicinity of transcription start sites of several genes [10], and the methylation status of these regulatory DNA sequences correlates with the transcriptional activity of the corresponding genes [7].

The findings of an aberrant expression of normally silenced retrotransposable L1 elements in RA but not OA tissues, and their inducibility by treatment of cultured SF with the demethylating agent 5-aza-2'-deoxycytidine, suggest a functional role for DNA hypomethylation in the pathogenesis of RA [11]. Based on this first indication, members of our group detected a global DNA hypomethylation in RASF and RA tissues, and normal SF cultured in a hypomethylating milieu acquired an activated phenotype similar to RASF [12]. The global DNA hypomethylation was shown to be due to an increased polyamine metabolism in RASF, resulting in decreased levels of $S$-adenosyl-L-methionine [13]. Furthermore, the expression of DNMT1 protein was shown to be decreased, accompanied by an increased expression of the proliferation marker proliferating cell nuclear antigen, in RASF compared with OASF [12]. A global DNA hypomethylation was also shown in peripheral blood mononuclear cells (PBMC) derived from RA patients compared with healthy controls. In RA-derived PBMC, however, the mRNA expression levels of DNMT1 were increased [14]. Besides global changes of DNA methylation patterns in RA, hypomethylation of promoter regions of single genes, such as for chemokine (C-X-C motif) ligand 12 (CXCL-12) were reported [15]. Recent publications demonstrated that even demethylation of a single $\mathrm{CpG}$ motif in the promoters of the $I L-6[16,17]$ and $I L-10$ $[18,19]$ genes correlated with their expression levels, and therefore contributed to elevated cytokine levels during disease. On the other hand, some DNA regions can also be hypermethylated in RA. The promoter region of the death receptor 3 gene (DR3), a member of the apoptosis inducing Fas gene family, was shown to be hypermethylated in RA patients [20].

DNA methylation was also shown to regulate expression levels of miRNAs [21,22]. miRNAs are a class of short noncoding RNAs that function as post-translational repressors of gene expression by binding to the 3' untranslated region of target genes. This leads to either degradation and/or inhibition of translation [23]. A screening of 260 individual miRNAs for differential 


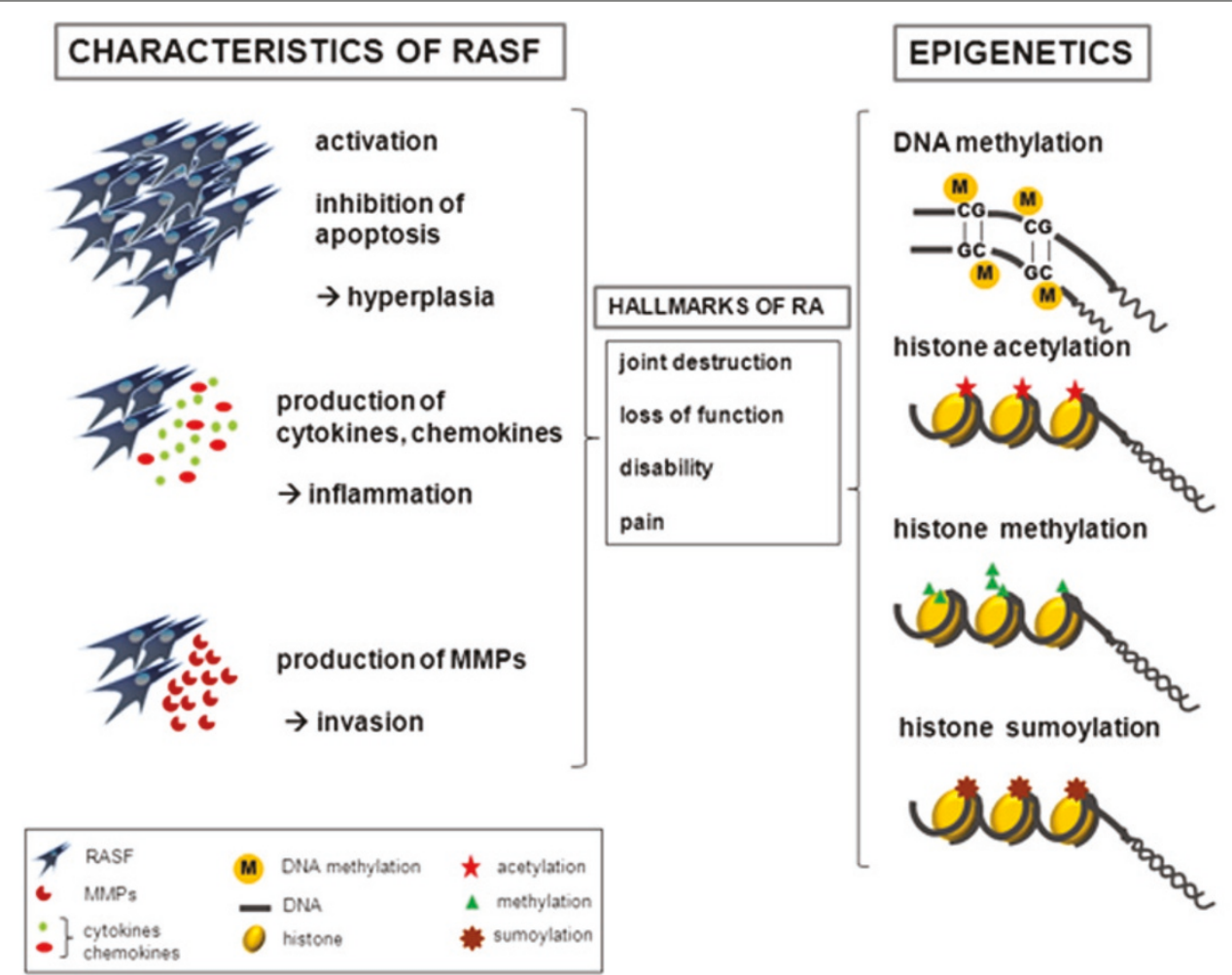

Figure 1. Epigenetic modifications in rheumatoid arthritis synovial fibroblasts contribute to their aggressive phenotype. Rheumatoid arthritis synovial fibroblasts (RASF) are characterized by excessive activation and an apoptosis-resistant phenotype, leading to hyperplasia of the synovium. Furthermore, RASF are capable of producing chemokines and cytokines, and thereby promote inflammation. The production of matrix metalloproteinases (MMPs) leads to invasion of RASF into cartilage. These characteristics of RASF contribute to the hallmarks of RA, namely joint destruction, loss of function, disability and pain. Changes in epigenetic modifications, such as DNA methylation, histone acetylation, histone methylation, and histone sumoylation, were described in pathologically activated RASF.

expression in RASF and OASF revealed that an array of miRNAs is overexpressed in RASF. One of the most differentially expressed miRNAs in RASF compared with OASF was miR-203. By treating RASF with the demethylating agent 5-aza-2'-deoxycytidine, miR-203 expression was further induced. Overexpression of miR-203 in RASF resulted in an increased expression of MMP1 as well as increased IL-6 secretion rates [21]. One miRNA that is downregulated in RA and regulated by methylation of the promoter region is miR-34a*. Decreased expression of miR-34a* in RASF resulted in upregulation of its direct target $\mathrm{X}$-linked inhibitor of apoptosis protein, and thereby contributed to the apoptosis resistant phenotype of RASF [22]. The future role of miRNAs in the pathogenesis of RA was recently reviewed by our laboratory [24].

Another study investigating the involvement of DNA methylation in the pathogenesis of RA came from a group investigating the silencing of the second $\mathrm{X}$ chromosome in females. This study revealed an increased presence of a skewed XCI pattern in RA patients. Instead of a random XCI, at least $80 \%$ of cells exhibited an inactivation of the same X chromosome [25]. These data suggest a role for XCI mosaicism in the pathogenesis of RA and might be one of the explanations for why RA is more prevalent in women.

\section{Histone acetylation and deacetylation}

The best studied histone modifications are the acetylation of specific lysine residues of histones $\mathrm{H} 3$ and H4. These modifications play a fundamental role in transcriptional regulation but also other processes such as replication. The acetylation state of a chromatin region is tightly controlled by two antagonizing enzyme families, namely histone acetylase transferases (HATs) and histone deacetylases (HDACs) [26]. Acetylation is generally associated with transcriptional activation. HATs such as lysine acetyltransferase 2A (GCN5), lysine acetyltransferase $2 \mathrm{~B}$ (PCAF), CREB binding protein, p300, and lysine acetyltransferase 8 (MOF) often function as transcriptional co-activators. On the contrary, deacetylation of chromatin is linked to gene silencing and HDACs were identified as transcriptional co-repressors. In humans, 18 HDACs have been identified and they are further 
subgrouped into four classes based on their orthologous homology to yeast (Table 1). Class I HDACs include HDAC1, HDAC2, HDAC3 and HDAC8, whereas the group of class II HDACs comprises HDAC4, HDAC5, HDAC6, HDAC7, HDAC9 and HDAC10. The seven sirtuins SIRT1 to SIRT7 build up the group of class III HDACs. HDAC11 is the only human enzyme classified as a class IV HDAC [26].

Most of the available data about the role of HDACs in the pathogenesis of RA were obtained using HDAC inhibitors (Table 1), first and foremost trichostatin A (TSA), an inhibitor of all class I and class II HDACs [27]. The lack of HDAC-specific inhibitors makes the interpretation of data difficult. Furthermore, one must bear in mind that HATs and HDACs target not only histones but also other proteins including transcription factors such as NF- $\mathrm{kB}$ and signal transducer and activator of transcription 3 . These transcription factors also play key roles in inflammatory processes and are therefore also involved in pathogenic processes in RA.

TSA was shown to sensitize RASF for TNF $\alpha$-related apoptosis-inducing ligand-induced apoptosis and to induce a cell cycle arrest by inducing the cell cycle regulator p21 [28]. A positive effect of the HDAC inhibitor FK228 on inhibiting RASF proliferation by induction of $\mathrm{p} 21$ and $\mathrm{p} 16$ promoter acetylation and expression was previously shown by Nishida and colleagues [29]. High doses of TSA and nicotinamide, a SIRT inhibitor, were also effective in inducing apoptosis in RA macrophages [30]. HDAC inhibitors might thus positively influence synovial hyperplasia in RA, on the one hand by inhibiting the activation of RASF and on the other by inducing apoptosis in macrophages.

Furthermore, these inhibitors also reduced the TNF $\alpha$ induced production of IL- 6 and the lipopolysaccharideinduced secretion of IL-6 and TNF $\alpha$. Interestingly, the authors could not find a close association between the beneficial effects of HDAC inhibitors on cytokine production and the overall HDAC inhibitor-dependent histone $\mathrm{H} 3$ and $\mathrm{H} 4$ acetylation [30]. These results indicate that not all effects of HDAC inhibitors are related to histone modifications. Recently, Grabiec and colleagues identified one of the molecular mechanisms underlying the anti-inflammatory activity of HDAC inhibitors. The authors demonstrated that the HDAC inhibitors TSA and ITF2357 suppressed cytokine-induced and Toll-like receptor ligand-induced expression of IL- 6 in RASF and macrophages by accelerating IL-6 mRNA decay. However, also other, not yet identified molecular mechanisms are involved in beneficial effects of HDAC inhibitors in RA, since the authors also stated that reduced mRNA stability did not explain the suppressive effects of cytokine-induced MMP1 and IL-8 expression in RASF [31].
Beneficial effects of HDAC inhibitors have been demonstrated in vivo by several groups using animal models of arthritis [29,32-35]. Since then, specific HDAC inhibitors have been continuously discussed as potential therapeutics not only in cancer but also in RA. Systemic administration of the HDAC inhibitor FK228 reduced joint swelling, synovial inflammation and subsequent bone and cartilage destruction in a mouse model of autoantibody-mediated arthritis [29]. The prophylactic and therapeutic effects of the HDAC inhibitors suberoylanilide hydroxamic acid and MS-275 were addressed in collagen-induced arthritis in rats and mice. This study also led to the conclusion that HDAC inhibitors are an innovative therapeutic strategy for RA [32]. In the same model, a dose-dependent effect of TSA was shown to reduce clinical signs of arthritis. The authors detected an increased number of cells with acetylated histone $\mathrm{H} 4$. Also, tissue inhibitor of MMP1 (TIMP1)-positive cells were increased, while the numbers of MMP3-positive and MMP13-positive chondrocytes were reduced as detected by immunohistochemical staining [33]. Furthermore, the HDAC inhibitor valproic acid significantly decreased disease incidence and severity in collageninduced arthritis mice. These effects were mediated by improving the production and function of $\mathrm{CD} 25^{+} \mathrm{FOXP3}{ }^{+}$ regulatory $\mathrm{T}$ cells, whose function is defective in RA patients [34].

Although much data on beneficial effects of HDAC inhibitors have been reported, the role of individual HDACs in the development of RA and their functional role in SF have not been fully elucidated to date and are still a matter of debate. In analogy to the investigation of global changes of DNA methylation in RA tissues and RASF, research groups also aimed to verify potential global changes in histone acetylation in RA. Initially, diminished HDAC1 and HDAC2 expression and a reduced total HDAC activity were described in synovial tissues of RA compared with OA patients, implicating a shift towards histone hyperacetylation in patients with RA and pointing to a potential role of HDACs in the pathogenesis of RA [36]. Later, an increased expression of HDAC1 and HDAC2 in RASF compared with OASF was described. By silencing these enzymes in RASF, the authors could demonstrate a functional role of HDAC1 and HDAC2 on proliferation and susceptibility to apoptosis [37].

Kawabata and colleagues also investigated the total HDAC activity in nuclear extracts of OA and RA tissues. Controversial to previous reports, in this study an increased HDAC activity and an unchanged HAT activity in RA tissues compared with OA tissues was reported. Furthermore, a decreased mRNA expression of HDAC4 and an increased mRNA and protein expression of HDAC1 in RA tissues were shown. Interestingly, 
Table 1. Classification of histone deacetylases and their inhibitors

\begin{tabular}{llll}
\hline Class & Member & Inhibitors & References \\
\hline I & HDAC1 & TSA, SAHA, ITF2357, FK228, VPA, MS-275 & {$[27,40,47-51]$} \\
& HDAC2 & TSA, SAHA, ITF2357, FK228, VPA \\
& HDAC3 & TSA, SAHA, ITF2357, FK228, VPA, MS-275, MI192 \\
& HDAC8 & TSA, SAHA, ITF2357, FK228, VPA \\
& HDAC4 & TSA, SAHA, ITF2357, VPA \\
& HDAC5 & TSA, SAHA, ITF2357, VPA \\
& HDAC6 & TSA, SAHA, ITF2357, VPA \\
& HDAC7 & TSA, SAHA, ITF2357, VPA \\
& HDAC9 & TSA, SAHA, ITF2357, VPA \\
& HDAC10 & TSA, SAHA, ITF2357, VPA \\
III & SIRT1 to SIRT7 & Nicotinamide \\
\hline
\end{tabular}

Human histone deacetylases (HDACs) were classified into four subgroups. HDAC inhibitors summarized in the text are listed. SAHA, suberoylanilide hydroxamic acid; SIRT, sirtuin; TSA, trichostatin A; VPA, valproic acid.

stimulation of RASF with TNF $\alpha$ specifically increased total HDAC activity and the expression of HDAC1 [38], suggesting that changes in synovial HDAC activity and expression may be influenced by the local inflammatory status [39].

A total increased HDAC activity was also reported recently in PBMC derived from RA patients, irrespective of whether or not patients were treated with the TNF $\alpha$ inhibitor etanercept. TSA and the HDAC3 selective inhibitor MI192 reduced lipopolysaccharide-stimulated cytokine production in PBMC derived from healthy donors and RA patients in a cell-type and compoundspecific manner [40]. The significance of studies analyzing HDAC expression levels is currently quite unclear, since the environmental influences that lead to the variability in HDAC expression and activity in synovial tissues are far from being understood.

At present, only few reports exist showing specific changes in promoter histone acetylation in RA [29,41]. Stimulation of RASF with TNF $\alpha$ and IL-1 $\beta$ induced the promoter acetylation of the cell cycle regulator p21 and correlated with RASF proliferation [29]. Furthermore, the promoter region of MMP1 was shown to be hyperacetylated in RASF and the authors could show a functional role for HDAC4 in this mechanism (see below) [41], being in line with the results from Kawabata and colleagues reporting a reduced HDAC4 expression in RA tissues [38].

The functional role of SIRTs in RA has been rarely studied to date $[30,42]$. As already mentioned, Grabiec and colleagues showed a positive effect of SIRT inhibitors in macrophages [30]. Recently, SIRT1 was reported to be overexpressed in RASF and monocytes derived from RA patients and expression levels of SIRT1 were further increased in the presence of TNF $\alpha$. Overexpression of SIRT1 contributed to the apoptosis resistance in RASF and promoted the proinflammatory cytokine production in monocytes [42]. The functional role of other members of the SIRT family has not yet been studied.

\section{Histone methylation}

Data on the contribution of histone modifications other than acetylation in the pathogenesis of RA are still rare, and the role of epigenetic methylation marks on lysine and/or arginine residues of histones is far less understood than the function of histone acetylation. The predominant histones modified by methylation are histones $\mathrm{H} 3$ and H4. Lysine residues can be monomethylated, dimethylated or trimethylated, adding yet another layer of complexity to this histone mark [8]. Depending on the methylated position, methylation of histones can be associated with more active or inactive chromatin regions. The trimethylation of histone 3 lysines $\mathrm{H} 3 \mathrm{~K} 27 \mathrm{~m} 3$, $\mathrm{H} 3 \mathrm{~K} 9 \mathrm{~m} 3$ and $\mathrm{H} 4 \mathrm{~K} 20 \mathrm{~m} 3$ are generally connected to transcriptionally silenced genes, whereas H3K36m3, $\mathrm{H} 3 \mathrm{~K} 4 \mathrm{~m} 3$ and H3K79m3 are associated with an open chromatin structure and transcriptionally active genes [43].

Our group addressed for the first time the role of histone methylation in RA and described an overexpression of the histone methyltransferase enhancer of zeste homologue 2 (EZH2) in RASF compared with OASF. EZH2 is the catalytic subunit of the polycomb repressor complex 2 that adds up to three methyl marks to H3K27 of genes designated for silencing [44]. EZH2 overexpression resulted in a reduced expression of the secreted frizzeled-related protein 1 (SFRP1), an inhibitor of the wingless-type MMTV integration site (Wnt) 
signaling pathway, which plays an important role in joint development and has been implicated in the pathogenesis of inflammatory arthropathies. However, the described mechanism seems to be specific for SFRP1 and elevated EZH2 expression levels did not correlate with global changes in H3K27me3 in RASF [45].

\section{Sumoylation}

Another post-translational modification described in RA is sumoylation [46]. Members of our group showed that RASF have intrinsically high levels of the small ubiquitinlike modifier 1 paralleled by decreased levels of its specific protease SENP1. By overexpressing SENP1 in RASF, histone $\mathrm{H} 4$ acetylation in the MMP1 promoter was reduced, leading to a reduced invasiveness of RASF. The authors showed that SENP1 overexpression is also accompanied by a global reduction of histone $\mathrm{H} 4$ acetylation and that the mechanism depends on the presence of HDAC4 [41].

\section{Conclusions}

There is no doubt that epigenetic modifications affect RA. Several studies demonstrated that epigenetics contribute to the aggressive, intrinsic activated phenotype of RASF and also the first in vivo data point to the beneficial effects of drugs modifying epigenetic patterns in animal models. However, more data are needed to clarify which modifications are causative for the disease onset and which epigenetic changes occur during chronic disease states. Whereas the picture of a differential pattern of DNA methylation in RA becomes clearer, other epigenetic modifications in RA have not yet been studied at all, or their functional role in the pathogenesis of RA is still puzzling. Understanding the complexity of different epigenetic modifications that regulate gene expression in cooperation and understanding the influence of environmental factors and current therapies on epigenetic modifications is just at the beginning. The available data raise the hope that, in future, detailed knowledge of epigenetic regulatory mechanisms in RA will help to develop new treatment strategies or will predict treatment efficacy and treatment outcome.

This article is part of the series on Epigenetics and rheumatic diseases, edited by Nan Shen. Other articles in this series can be found at http://arthritis-research.com/series/epigenetics

\footnotetext{
Abbreviations

ACPA, antibodies against citrullinated peptide antigens; $C p G$, cytosinephosphate-guanine dinucleotide; DNMT, DNA methyltransferase; EZH2, enhancer of zeste homologue 2; H3K, histone 3 lysine; HAT, histone acetylase transferase; HDAC, histone deacetylase; IL, interleukin; miRNA, microRNA; MMP matrix metalloproteinase; NF-KB, nuclear factor kappa-light-chain-enhancer of activated $\mathrm{B}$ cells; $\mathrm{OA}$, osteoarthritis; PBMC, peripheral blood mononuclear cells; RA, rheumatoid arthritis; SENP1, small ubiquitin-like modifier 1-specific protease 1; SF, synovial fibroblasts; SFRP1, secreted frizzeled-related protein 1;
}

SIRT, sirtuin; TNF, tumor necrosis factor; TSA, trichostatin A; Wnt, wingless-type MMTV integration site; $\mathrm{XCI}, \mathrm{X}$-chromosome inactivation.

\section{Competing interests}

The authors declare that they have no competing interests.

\section{Acknowledgements}

This research is supported by FP7 Masterswitch, IMI-BT Cure, and IAR Epalinges.

Published: 9 November 2012

\section{References}

1. Smolen J, Aletaha D: The burden of rheumatoid arthritis and access to treatment: a medical overview. Eur J Health Econ 2008, 8(Suppl 2):S39-S47.

2. Padyukov L, Seielstad M, Ong RT, Ding B, Ronnelid J, Seddighzadeh M, Alfredsson $L$, Klareskog $L$ : A genome-wide association study suggests contrasting associations in ACPA-positive versus ACPA-negative rheumatoid arthritis. Ann Rheum Dis 2011, 70:259-265.

3. Saevarsdottir $S$, Wallin H, Seddighzadeh M, Ernestam S, Geborek P, Petersson IF, Bratt J, van Vollenhoven RF: Predictors of response to methotrexate in early DMARD naive rheumatoid arthritis: results from the initial open-label phase of the SWEFOT trial. Ann Rheum Dis 2011, 70:469-475.

4. Lefèvre S, Knedla A, Tennie C, Kampmann A, Wunrau C, Dinser R, Korb A, Schnaker EM, Tarner IH, Robbins PD, Evans CH, Stürz H, Steinmeyer J, Gay S, Schölmerich J, Pap T, Müller-Ladner U, Neumann E: Synovial fibroblasts spread rheumatoid arthritis to unaffected joints. Nat Med 2009, 15:1414-1420.

5. Ospelt C, Gay S: The role of resident synovial cells in destructive arthritis. Best Pract Res Clin Rheumatol 2008, 22:239-252.

6. Bird A: Perceptions of epigenetics. Nature 2007, 447:396-398.

7. Turek-Plewa J, Jagodzinski PP: The role of mammalian DNA methyltransferases in the regulation of gene expression. Cell Mol Biol Lett 2005, 10:631-647.

8. Strahl $B D, A$ Allis CD: The language of covalent histone modifications. Nature 2000, 403:41-45

9. Suzuki MM, Bird A: DNA methylation landscapes: provocative insights from epigenomics. Nat Rev Genet 2008, 9:465-476.

10. Jones PA: Functions of DNA methylation: islands, start sites, gene bodies and beyond. Nat Rev Genet 2012, 13:484-492.

11. Neidhart M, Rethage J, Kuchen S, Kunzler P, Crowl RM, Billingham ME, Gay RE, Gay S: Retrotransposable L1 elements expressed in rheumatoid arthritis synovial tissue: association with genomic DNA hypomethylation and influence on gene expression. Arthritis Rheum 2000, 43:2634-2647.

12. Karouzakis E, Gay RE, Michel BA, Gay S, Neidhart M: DNA hypomethylation in rheumatoid arthritis synovial fibroblasts. Arthritis Rheum 2009, 60:3613-3622.

13. Karouzakis E, Gay RE, Gay S, Neidhart M: Increased recycling of polyamines is associated with global DNA hypomethylation in rheumatoid arthritis synovial fibroblasts. Arthritis Rheum 2012, 64:1809-1817.

14. Liu CC, Fang TJ, Ou TT, Wu CC, Li RN, Lin YC, Lin CH, Tsai WC, Liu HW, Yen JH: Global DNA methylation, DNMT1, and MBD2 in patients with rheumatoid arthritis. Immunol Lett 2011, 135:96-99.

15. Karouzakis E, Rengel Y, Jungel A, Kolling C, Gay RE, Michel BA, Tak PP, Gay S, Neidhart M, Ospelt C: DNA methylation regulates the expression of CXCL12 in rheumatoid arthritis synovial fibroblasts. Genes Immun 2011, 12:643-652.

16. Nile CJ, Read RC, Akil M, Duff GW, Wilson AG: Methylation status of a single CpG site in the IL6 promoter is related to IL6 messenger RNA levels and rheumatoid arthritis. Arthritis Rheum 2008, 58:2686-2693.

17. Ishida K, Kobayashi T, Ito S, Komatsu Y, Yokoyama T, Okada M, Abe A, Murasawa A, Yoshie H: Interleukin-6 gene promoter methylation in rheumatoid arthritis and chronic periodontitis. J Periodontol 2012, 83:917-925.

18. Fu LH, Ma CL, Cong B, Li SJ, Chen HY, Zhang JG: Hypomethylation of proximal $\mathrm{CpG}$ motif of interleukin-10 promoter regulates its expression in human rheumatoid arthritis. Acta Pharmacol Sin 2011, 32:1373-1380.

19. Lin SY, Hsieh SC, Lin YC, Lee CN, Tsai MH, Lai LC, Chuang EY, Chen PC, Hung CC, Chen LY, Hsieh WS, Niu DM, Su YN, Ho HN: A whole genome methylation analysis of systemic lupus erythematosus: hypomethylation of the IL10 and IL1R2 promoters is associated with disease activity. Genes Immun 2012, 13:214-220 
20. Takami N, Osawa K, Miura Y, Komai K, Taniguchi M, Shiraishi M, Sato K, Iguchi T, Shiozawa K, Hashiramoto A, Shiozawa S: Hypermethylated promoter region of DR3, the death receptor 3 gene, in rheumatoid arthritis synovial cells. Arthritis Rheum 2006, 54:779-787.

21. Stanczyk J, Ospelt C, Karouzakis E, Filer A, Raza K, Kolling C, Gay R, Buckley CD, Tak PP, Gay S, Kyburz D: Altered expression of microRNA-203 in rheumatoid arthritis synovial fibroblasts and its role in fibroblast activation. Arthritis Rheum 2011, 63:373-381.

22. Niederer F, Trenkmann M, Ospelt C, Karouzakis E, Neidhart M, Stanczyk Kolling C, Gay RE, Detmar M, Gay S, Jüngel A, Kyburz D: Downregulation of microRNA-34a* in rheumatoid arthritis synovial fibroblasts promotes apoptosis resistance. Arthritis Rheum 2012, 64:1771-1779.

23. Bartel DP: MicroRNAs: genomics, biogenesis, mechanism, and function Cell 2004, 116:281-297.

24. Filkova M, Jungel A, Gay RE, Gay S: MicroRNAs in rheumatoid arthritis: potential role in diagnosis and therapy. BioDrugs 2012, 26:131-141.

25. Chabchoub G, Uz E, Maalej A, Mustafa CA, Rebai A, Mnif M, Bahloul Z, Farid NR, Ozcelik T, Ayadi H: Analysis of skewed X-chromosome inactivation in females with rheumatoid arthritis and autoimmune thyroid diseases. Arthritis Res Ther 2009, 11:R106.

26. Wang Z, Zang C, Cui K, Schones DE, Barski A, Peng W, Zhao K: Genome-wide mapping of HATs and HDACs reveals distinct functions in active and inactive genes. Cell 2009, 138:1019-1031.

27. Vigushin DM, Ali S, Pace PE, Mirsaidi N, Ito K, Adcock I, Coombes RC: Trichostatin A is a histone deacetylase inhibitor with potent antitumor activity against breast cancer in vivo. Clin Cancer Res 2001, 7:971-976.

28. Jungel A, Baresova V, Ospelt C, Simmen BR, Michel BA, Gay RE, Gay S, Seemayer CA, Neidhart M: Trichostatin A sensitises rheumatoid arthritis synovial fibroblasts for TRAIL-induced apoptosis. Ann Rheum Dis 2006 65:910-912

29. Nishida K, Komiyama T, Miyazawa S, Shen ZN, Furumatsu T, Doi H, Yoshida A, Yamana J, Yamamura M, Ninomiya Y, Inoue H, Asahara H: Histone deacetylase inhibitor suppression of autoantibody-mediated arthritis in mice via regulation of $\mathrm{p} 16 \mathrm{INK} 4 \mathrm{a}$ and $\mathrm{p} 21$ (WAF1/Cip1) expression. Arthritis Rheum 2004, 50:3365-3376.

30. Grabiec AM, Krausz S, de Jager W, Burakowski T, Groot D, Sanders ME, Prakken BJ, Maslinski W, Eldering E, Tak PP, Reedquist KA: Histone deacetylase inhibitors suppress inflammatory activation of rheumatoid arthritis patient synovial macrophages and tissue. J Immuno/ 2010, 184:2718-2728.

31. Grabiec AM, Korchynskyi O, Tak PP, Reedquist KA: Histone deacetylase inhibitors suppress rheumatoid arthritis fibroblast-like synoviocyte and macrophage IL-6 production by accelerating mRNA decay. Ann Rheum Dis 2012, 71:424-431

32. Lin HS, Hu CY, Chan HY, Liew YY, Huang HP, Lepescheux L, Bastianelli E, Baron R, Rawadi G, Clement-Lacroix P: Anti-rheumatic activities of histone deacetylase (HDAC) inhibitors in vivo in collagen-induced arthritis in rodents. Br J Pharmacol 2007, 150:862-872.

33. Nasu Y, Nishida K, Miyazawa S, Komiyama T, Kadota Y, Abe N, Yoshida A, Hirohata S, Ohtsuka A, Ozaki T: Trichostatin A, a histone deacetylase inhibitor, suppresses synovial inflammation and subsequent cartilage destruction in a collagen antibody-induced arthritis mouse model. Osteoarthritis Cartilage 2008, 16:723-732.

34. Saouaf SJ, Li B, Zhang G, Shen Y, Furuuchi N, Hancock WW, Greene MI: Deacetylase inhibition increases regulatory $T$ cell function and decreases incidence and severity of collagen-induced arthritis. Exp Mol Pathol 2009, 87:99-104.

35. Joosten LA, Leoni F, Meghii S, Mascagni P: Inhibition of HDAC activity by ITF2357 ameliorates joint inflammation and prevents cartilage and bone destruction in experimental arthritis. Mol Med 2011, 17:391-396.

36. Huber LC, Brock M, Hemmatazad H, Giger OT, Moritz F, Trenkmann M, Distler $J$ H, Gay RE, Kolling C, Moch H, Michel BA, Gay S, Distler O, Jüngel A: Histone deacetylase/acetylase activity in total synovial tissue derived from rheumatoid arthritis and osteoarthritis patients. Arthritis Rheum 2007, 56:1087-1093.
37. Horiuchi M, Morinobu A, Chin T, Sakai Y, Kurosaka M, Kumagai S: Expression and function of histone deacetylases in rheumatoid arthritis synovial fibroblasts. J Rheumatol 2009, 36:1580-1589.

38. Kawabata T, Nishida K, Takasugi K, Ogawa H, Sada K, Kadota Y, Inagaki J, Hirohata S, Ninomiya Y, Makino H: Increased activity and expression of histone deacetylase 1 in relation to tumor necrosis factor-alpha in synovial tissue of rheumatoid arthritis. Arthritis Res Ther 2010, 12:R133.

39. Grabiec AM, Reedquist KA: Histone deacetylases in RA: epigenetics and epiphenomena. Arthritis Res Ther 2010, 12:142.

40. Gillespie J, Savic S, Wong C, Hempshall A, Inman M, Emery P, Grigg R, McDermott MF: Histone deacetylases are dysregulated in rheumatoid arthritis and a novel histone deacetylase 3-selective inhibitor reduces interleukin- 6 production by peripheral blood mononuclear cells from rheumatoid arthritis patients. Arthritis Rheum 2012, 64:418-422.

41. Maciejewska-Rodrigues $H$, Karouzakis E, Strietholt S, Hemmatazad H, Neidhart M, Ospelt C, Gay RE, Michel BA, Pap T, Gay S, Jüngel A: Epigenetics and rheumatoid arthritis: the role of SENP1 in the regulation of MMP-1 expression. J Autoimmun 2010, 35:15-22.

42. Niederer F, Ospelt C, Brentano F, Hottiger MO, Gay RE, Gay S, Detmar M, Kyburz D: SIRT1 overexpression in the rheumatoid arthritis synovium contributes to proinflammatory cytokine production and apoptosis resistance. Ann Rheum Dis 2011, 70:1866-1873.

43. Kouzarides T: Chromatin modifications and their function. Cell 2007, 128:693-705.

44. Margueron R, Reinberg D: The Polycomb complex PRC2 and its mark in life. Nature 2011, 469:343-349.

45. Trenkmann M, Brock M, Gay RE, Kolling C, Speich R, Michel BA, Gay S, Huber LC: Expression and function of EZH2 in synovial fibroblasts: epigenetic repression of the Wnt inhibitor SFRP1 in rheumatoid arthritis. Ann Rheum Dis 2011, 70:1482-1488

46. Meinecke I, Cinski A, Baier A, Peters MA, Dankbar B, Wille A, Drynda A, Mendoza H, Gay RE, Hay RT, Ink B, Gay S,Pap T: Modification of nuclear PML protein by SUMO-1 regulates Fas-induced apoptosis in rheumatoid arthritis synovial fibroblasts. Proc Natl Acad Sci U S A 2007, 104:5073-5078.

47. Marks PA, Breslow R: Dimethyl sulfoxide to vorinostat: development of this histone deacetylase inhibitor as an anticancer drug. Nat Biotechnol 2007, 25:84-90.

48. Furumai R, Matsuyama A, Kobashi N, Lee KH, Nishiyama M, Nakajima $H_{\text {, }}$ Tanaka A, Komatsu Y, Nishino N, Yoshida M, Horinouchi S: FK228 (depsipeptide) as a natural prodrug that inhibits class I histone deacetylases. Cancer Res 2002, 62:4916-4921.

49. Golay J Cuppini L, Leoni F, Mico C, Barbui V Domenghini M, Lombardi L, Ner A, Barbui AM, Salvi A, Pozzi P,Porro G, Pagani P, Fossati G, Mascagni P, Introna M, Rambaldi A: The histone deacetylase inhibitor ITF2357 has antileukemic activity in vitro and in vivo and inhibits IL-6 and VEGF production by stromal cells. Leukemia 2007, 21:1892-1900.

50. Gottlicher M, Minucci S, Zhu P, Kramer OH, Schimpf A, Giavara S, Sleeman JP, Lo Coco F, Nervi C, Pelicci PG, Heinzel T: Valproic acid defines a novel class of HDAC inhibitors inducing differentiation of transformed cells. EMBO J 2001, 20:6969-6978

51. Saito A, Yamashita T, Mariko Y, Nosaka Y, Tsuchiya K, Ando T, Suzuki T, Tsuruo T, Nakanishi O: A synthetic inhibitor of histone deacetylase, MS-27-275, with marked in vivo antitumor activity against human tumors. Proc Natl Acad Sci USA 1999, 96:4592-4597.

52. Giammona LM, Panuganti S, Kemper JM, Apostolidis PA, Lindsey S, Papoutsakis ET, Miller WM: Mechanistic studies on the effects of nicotinamide on megakaryocytic polyploidization and the roles of NAD+ levels and SIRT inhibition. Exp Hemato/ 2009, 37:1340-1352, e1343.

\section{doi:10.1186/ar4074}

Cite this article as: Klein K, et al:: Epigenetic contributions in the development of rheumatoid arthritis. Arthritis Research \& Therapy 2012, $14: 227$. 